\title{
THREE SEQUENTIAL BRAIN ACTIVATIONS ENCODE MENTAL TRANSFORMATIONS OF UPRIGHT AND INVERTED HUMAN BODIES: A HIGH RESOLUTION EVOKED POTENTIAL STUDY
}

\author{
T. TADI, ${ }^{a}$ L. S. OVERNEYa AND O. BLANKE ${ }^{a, b *}$ \\ aLaboratory of Cognitive Neuroscience, Brain-Mind Institute, École \\ Polytechnique Federale de Lausanne, 1015 Lausanne, Switzerland \\ ${ }^{b}$ Department of Neurology, University Hospital, Geneva, Switzerland
}

\begin{abstract}
Human bodies provide a particularly rich source of visual information. Whereas most previous studies have focused on the neural mechanisms during the perception and recognition of human bodies, the aim of the present study was to investigate the time course and location of brain activation during mental imagery of human bodies. When participants were asked to imagine themselves in the position of a visually presented human body as seen from many different angles and at two orientations (upright or inverted), their reaction times were faster for upright as compared to inverted bodies and correlated differently with the tested angles. These behavioral effects were also reflected in brain activation patterns, but only during the time period from 220 to $490 \mathrm{~ms}$ after stimulus onset. Evoked potential mapping and electrical neuroimaging revealed three distinct and sequential steps of processing related to mental body transformation: (1) an early activation in temporo-occipital and temporo-parietal cortex (220-360 ms) that does not distinguish between upright and inverted bodies, but closely reflects the effort of mental transformation, followed (2) by an activation in temporo-occipital and medial parieto-occipital cortex (350-460 ms) that encodes mental transformation for upright bodies, and (3) a later activation in temporo-occipital and prefrontal cortex (390-490 ms) that encodes mental transformation for inverted bodies. These data suggest that the mental transformation of human bodies is not a single process but a sequence of temporally distinct processing steps, where each step reflects a distinct aspect of the transformation process that consists of activations in a network of posterior brain areas including extrastriate cortex, temporoparietal cortex, and medial parieto-occipital cortex, as well as an anterior brain region in prefrontal cortex. (c) 2009 IBRO. Published by Elsevier Ltd. All rights reserved.
\end{abstract}

Key words: mental body imagery, body inversion, EP mapping, human neuroimaging.

Perceiving and recognizing the actions, moods, and intentions of other people are important social skills, and human bodies provide a particularly rich source of visual information in support of these abilities. Recent neuroimaging

${ }^{*}$ Correspondence to: O. Blanke, Laboratory of Cognitive Neuroscience, Brain-Mind Institute, École Polytechnique Federale de Lausanne, Station 19, 1015 Lausanne, Switzerland. Tel: +41-21-6939621; fax: +41-21-6939625. E-mail address: olaf.blanke@epfl.ch (O. Blanke).

Abbreviations: EEG, electroencephalography; EP, evoked potential; ERP, event-related potential; GFP, global field power; LAURA, local auto-regressive average; RT, response time. research has unraveled several brain mechanisms involved in the visual perception and recognition of human bodies (Allison et al., 2000; Downing et al., 2001; Thierry et al., 2006; Urgesi et al., 2007) either of one's own or of others (Keenan et al., 2001; Sugiura et al., 2005). These studies revealed the existence of an extended cortical network encompassing extrastriate areas, temporo-parietal and parietal cortex, as well as medial and lateral frontal regions. Another line of research has studied mental imagery with respect to human bodies. Mental imagery or mental transformation is the ability to imagine the rotation of an object (or human body) in space and is essential in many everyday cognitive tasks such as spatial reasoning, action planning and problem solving (Shepard and Hurwitz, 1984; Corballis, 1997). Tasks that require mental transformations in space are generally referred to as "mental rotation" tasks, and these have repeatedly shown that when deciding on the congruency of a rotated object with reference to another, reaction times increase linearly with the increasing angle of rotation (Shepard and Metzler, 1971; Harris et al., 2000; Petit and Harris, 2005). This typical monotonic response time (RT) function is generally interpreted as indicating that the process of mentally rotating an object is analogous to the physical rotation of an object which is constrained by physics to move through continuous points in space. This holds for objects (such as letters, numbers, and cubes) and also for body-parts. However, in the latter case, additional factors such as biomechanical constraints have also been shown to influence mental transformations (Sekiyama, 1982; Parsons, 1987b; Petit et al., 2003; Reed et al., 2003). Parsons (1987a) showed that the time required to mentally simulate a movement is similar to the time taken to actually perform that movement or position change, if the orientations of the stimulus are familiar. This temporal similarity is not observed in the case of unfamiliar postures or body part positions (Overney et al., 2005; Petit and Harris, 2005; Ionta et al., 2007). Such body constraints may also affect mental imagery for full human bodies.

There have been a number of neuroimaging studies on the mental transformation of external objects (Kosslyn et al., 1994; Cohen et al., 1996; Tagaris et al., 1996, 1997; Pegna et al., 1997; Richter et al., 1997; Carpenter, 1999; Harris et al., 2000; Jordan et al., 2001; Vingerhoets et al., 2001; Gauthier and Tarr, 2002; Podzebenko et al., 2002; Harris and Miniussi, 2003). These revealed an activation of parietal cortex, either exclusively in the right hemisphere (Harris et al., 2000; Harris and Miniussi, 2003), the left hemisphere (Alivisatos and Petrides, 1997; Vingerhoets et al., 2001), or bilaterally (Carpenter et al., 1999). Studies measuring event-

0306-4522/09 \$ - see front matter @ 2009 IBRO. Published by Elsevier Ltd. All rights reserved.

doi:10.1016/j.neuroscience.2009.02.012 
related potentials (ERPs) during the mental transformation of external objects revealed a negative component located over parietal scalp electrodes (Peronnet and Farah, 1989; Wijers et al., 1989; Yoshino et al., 2000; Milivojevic et al., 2003). Using evoked potential mapping (EP mapping) and electrical neuroimaging, Pegna et al. (1997) found a topographic pattern (or EP map) between 400 and $600 \mathrm{~ms}$ that increased in duration for greater angles of rotation and was localized to right parieto-occipital cortex.

Fewer studies have investigated the neural mechanisms of mental transformations of body parts (Cooper and Shepard, 1975; Parsons, 1987b; Parsons et al., 1995; Kosslyn et al., 1998; Thayer et al., 2001; Overney et al., 2005; Thierry et al., 2006; Fiorio et al., 2007). The neural mechanisms of body part mental transformations have been dissociated from those of non-bodily objects and localized to left parietal cortex (Bonda et al., 1996; Kosslyn et al., 1998; Ganis et al., 2000). Using EP mapping and electrical neuroimaging, Overney and colleagues (Overney et al., 2005; Overney and Blanke, in press) found an EP map between 310 and $380 \mathrm{~ms}$ after stimulus onset, localized to the left medial parieto-occipital cortex, that increased monotonically with the angle of body part rotation.

Very few studies have investigated the neural mechanisms underlying the mental transformations of whole bodies (Parsons, 1987a; Zacks et al., 1999, 2002; Blanke et al., 2005). In his seminal study using simple line drawings of whole bodies, Parsons (1987a) showed that when presented with a picture of a body, observers imagine their own body as rotating to match the body in the picture (this has also been observed with body part stimuli). Using schematic line drawings of whole bodies and measuring functional magnetic resonance imaging (fMRI) during a mental body transformation task, Zacks et al. (1999) revealed increased activation in temporo-occipital and temporo-parietal cortex, with a left hemisphere predominance for upright bodies and a right-sided parietal-temporaloccipital activation for inverted bodies. Using line drawings and whole body mental transformations in combination with EP mapping and electrical neuroimaging, Blanke et al. (2005) and Arzy et al. (2006) showed that such body transformations activate temporo-parietal cortex during a time period $330-400 \mathrm{~ms}$ after stimulus onset and that this activation was not found during a lateralization task which did not require mental body transformation and when mentally rotating non-bodily objects (Blanke et al., 2005). This temporo-parietal activation at $330-400 \mathrm{~ms}$ was prolonged for larger angles of body transformations and depended on the type of imagined body transformation (Arzy et al., 2006). Next to the temporo-parietal cortex, Arzy et al. (2006) also reported the implication of extrastriate cortex in mental transformations of whole bodies, probably including among other regions the extrastriate body area (Downing et al., 2001; Astafiev et al., 2004).

Given the importance of neural body representations in the processing of social information, and the importance of mental transformation, next to perception, in the processing of human bodily stimuli, we investigated here the time course and location of brain activation for mental whole body transformations. Based on the different time periods of mental body transformations as suggested by previous EP research (Blanke et al., 2005; Overney et al., 2005; Arzy et al., 2006). Also, we investigated whether mental body transformation is a single brain process or a sequence of brain processes at the suggested latencies. For this we used three-dimensional human bodies presented across different angles, EP mapping, and electrical neuroimaging. In addition, we investigated if such brain activations depend on whether mental body transformations were performed in response to upright bodies (as classically tested and most often encountered in natural conditions) or inverted (unfamiliar) bodies, as has been tested for body parts (Overney et al., 2005; Petit and Harris, 2005; lonta et al., 2007). We predicted (1) a monotonic increase in reaction times and error rates with the angle of upright, but not inverted bodies, based on Parsons (1987a) observation that unfamiliar postures or body positions impair or modify mental body transformations. We further predicted (2) that extrastriate and/or temporo-parietal activation in the time period of 300-450 ms (Blanke et al., 2005; Arzy et al., 2006) would increase monotonically with the angle of upright bodies and that (3) the mental transformations of inverted bodies would - at least partly_be characterized by different neural mechanisms.

\section{EXPERIMENTAL PROCEDURES}

\section{Participants}

Fourteen healthy volunteers (all male, aged 19-25 years, mean \pm SD: $22.2 \pm 3.7$ years) participated in the experiment. All participants were right-handed, had normal or corrected-to-normal vision, and declared of having no history of neurological or psychiatric disorders. All participants gave written informed consent prior to inclusion in the study, which had been approved by the Ethical Committee of Lausanne (Switzerland).

\section{Stimuli}

3D humanoid body images were created using Autodesk Motionbuilder 7 (San Rafael, CA, USA). We created images as if rotated around the yaw axis or vertical axis of the humanoid figure (Fig. 1) leading to eight different angles of rotation. The angles used in the current study are $0^{\circ}, 45^{\circ}, 90^{\circ}, 135^{\circ}, 180^{\circ}, 225^{\circ}, 270^{\circ}, 315^{\circ}$. All eight humanoid figures were shown in the same simple visual scene which consisted of the 3D humanoid figure and a mesh. Either the right or left hand of the figure was marked, and participants indicated which hand was marked (Fig. 1a, upright stimuli; the correct responses for each task are indicated in the figure legend). All stimuli had the same spatial dimensions $\left(27.6^{\circ}\right)$. Stimuli appeared for $200 \mathrm{~ms}$ in the centre of the computer screen, preceded by a fixation cross and an inter-stimulus interval of 1500 ms. We created eight other identical stimuli but inverted their orientation (Fig. 1b; inverted stimuli; the correct responses for each task are indicated in the figure legend). The computer screen (Viewsonic Corp, CA, USA) and a distance $(1 \mathrm{~m})$ from the subject's eyes to the monitor were the same across both conditions.

\section{Procedure}

Across both upright and inverted conditions and for each stimulus presentation, participants were asked to imagine themselves in the position and orientation of the humanoid figure (Fig. 1) presented on a computer screen (Zacks et al., 1999; Blanke et al., 
a.

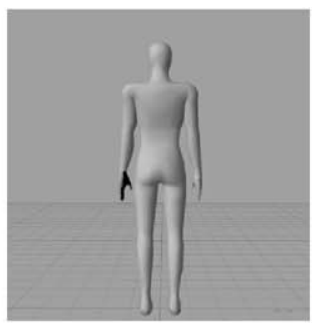

$0^{\circ}$

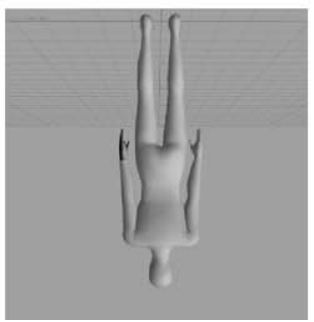

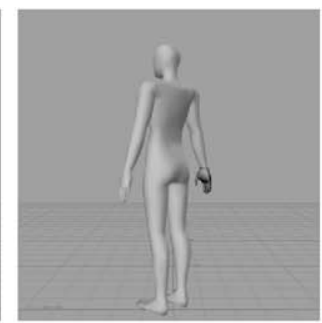

$45^{\circ}$

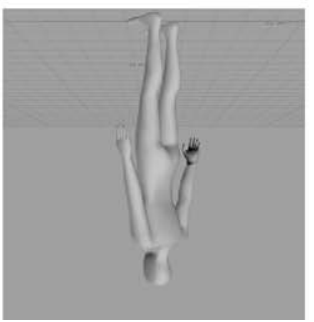

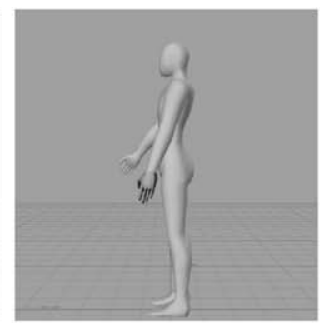

$90^{\circ}$

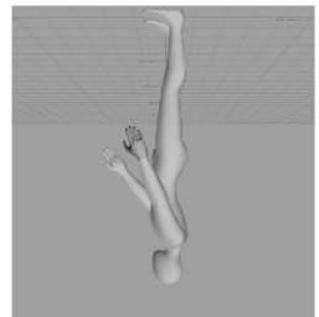

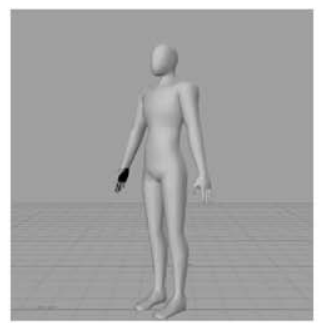

$135^{\circ}$

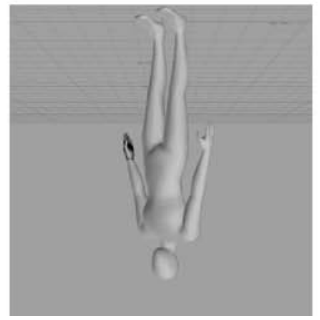

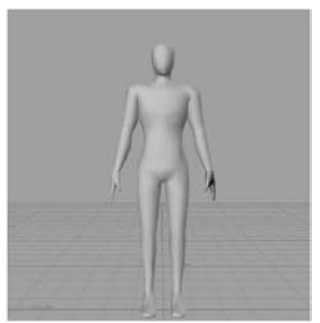

$180^{\circ}$

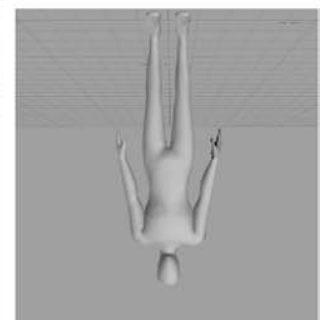

Fig. 1. (a) Upright stimuli with different angles of rotation $\left(0^{\circ}, 45^{\circ}, 90^{\circ}, 135^{\circ}, 180^{\circ}\right)$. Correct responses: left, right, left, right, left. (b) Inverted stimuli with different angles of rotation $\left(0^{\circ}, 45^{\circ}, 90^{\circ}, 135^{\circ}, 180^{\circ}\right)$. Correct responses: right, left, right, left, right.

2005; Zacks and Michelon, 2005). The participants were seated in an upright position on a chair with both their hands resting on a table in front of them. Their task was to indicate which hand was marked as has been classically used in tasks to assess mental transformation of body parts as well as whole bodies (Cooper and Shepard, 1975; Parsons, 1994; Corballis, 1997; Parsons et al., 1998; Zacks et al., 1999; Blanke et al., 2005; lonta et al., 2007). The task instructions were constant in both conditions. Responses were given via a button press on a serial response box. Responses were given using the index and middle finger. Five participants responded with the left hand and six participants with the right hand. The participants were instructed to respond as fast and precisely as possible but to always perform the requested mental imagery in both tasks before giving the response. Upright and inverted stimuli were presented in alternating fashion in a total of eight blocks (four blocks upright orientation; four blocks inverted orientation). Six of the participants began with an upright stimulus block and five participants with an inverted stimulus block. In every block, each stimulus appeared 10 times in randomized order giving rise to 160 visual presentations per block (eight angles with right hand marked; eight angles with left hand marked). Participants carried out practice trials presented at the start of the experiment for both the upright and inverted stimuli. In these practice blocks, each of the stimuli appeared once in randomized order. At the end of the experiment, subjective reports about their strategies were collected from all participants. For analysis, we collapsed the angles $45-315^{\circ}$, angles $135-225^{\circ}$, and angles $90-$ $270^{\circ}$ as the results were similar for these pairs of angles and they represent the same angle of rotation in opposite quadrants. Hence we will look at results for the five angles of rotation: $0^{\circ}, 45^{\circ}, 90^{\circ}$, $135^{\circ}, 180^{\circ}$ for both upright and inverted conditions (Fig. 1).

Task instructions. Across both upright and inverted conditions, participants were instructed with the following for each stimulus presentation: "imagine yourself in the position and orientation of the humanoid figure and indicate which hand is marked".

\section{Analysis of behavioral data}

We recorded RTs and error rates. Two $\times$ five repeated measures ANOVAs were run on RTs and error rates for correctly responded trials with orientation (upright vs. inverted) and angles of rotation $\left(0^{\circ}, 45^{\circ}, 90^{\circ}, 135^{\circ}, 180^{\circ}\right)$ as within subject factors. Of the 14 participants, one was excluded from the study due to extremely prolonged RTs (exceeding the mean by more than 3 standard deviations). Two further participants had to be excluded due to a highly artifacted electroencephalography (EEG). Hence, for the final analysis, we treated data from 11 participants.

\section{EEG recording and EP mapping}

Continuous EEG was acquired with a Biosemi system (Biosemi, Inc., USA) from 192 scalp electrodes (impedances $<50 \mathrm{k} \Omega$; vertex referenced; $2000 \mathrm{~Hz}$ digitization; band-pass filtered $0.1-200 \mathrm{~Hz}$ ) in a darkened, electrically shielded booth. Epochs of EEG (from 0 to 700 $\mathrm{ms}$ post-stimulus onset), only from trials yielding correct responses were averaged for each of the two experimental conditions and for each subject to calculate the EP. In addition to the rejection of sweeps where any channel exceeded the amplitude of $\pm 100 \mu \mathrm{V}$, the data were visually inspected to reject epochs with blinks, eye movements, or other sources of transient noise. The mean percentage of accepted epochs per condition was $80 \%$. For each subject's ERPs, artifacted channels were excluded and interpolated to a standard 192-channel electrode array. After this procedure and before group averaging, ERPs were band-pass filtered (1-40 Hz), baseline corrected and recalculated against the average reference.

As applied by many previous authors, the analysis of the 192-channel EPs was based on the examination of the spatial variations of the scalp voltage distribution over time and between conditions, an approach known as EP mapping (Lehmann et al., 1987; Pascual-Marqui et al., 1995; Michel et al., 1999, 2001, 2004; Itier and Taylor, 2004). This analysis is identical to our previous EP mapping work (Blanke et al., 2005; Overney et al., 2005; Arzy et al., 2006, 2007). This approach searches for time-segments of stable map topography that represent functional microstates of the brain during information processing. EP microstate segments were defined by using a spatial $k$-means cluster analysis to identify the dominant map topographies on the scalp in the groupaveraged EPs across the experimental conditions (upright and inverted) over time (Pascual-Marqui et al., 1995; Itier et al., 2004; Blanke et al., 2005). These maps are the mean maps over the period where the segment was found. The optimal number of these template maps is determined by a modified cross-validation 
criterion (Pascual-Marqui et al., 1995). In a second step the presence of a given EP map as identified in the group-averaged data is verified statistically in the EPs of the individual participants. This allows to determine the duration (number of time-points that were assigned to one microstate map) and the amplitude (or global field power, GFP) of a given EP map for each condition across participants. These values for a given EP map can then be subjected to statistical analysis. Statistical comparisons were performed on the duration (in ms) and amplitude GFP (in $\mu \mathrm{V}$ ) of each map (dependent variable) in the individual EPs using repeated measures ANOVAs, with the factors Orientation (upright vs. inverted) and Angle $\left(0^{\circ}, 45^{\circ}, 90^{\circ}, 135^{\circ}, 180^{\circ}\right)$. The amplitude of the GFP of each map is defined as the spatial root mean squared across all electrodes (Lehmann and Skrandies, 1980).

\section{Source localization}

The neural generators for a given mean EP map were estimated by using a distributed linear inverse solution, based on a local auto-regressive average (LAURA) model (Grave de Peralta Menendez et al., 2001, 2004) as used previously (Blanke et al., 2005; Arzy et al., 2006, 2007). LAURA selects the source configuration that tries to mimic the biophysical behavior of electric vector fields (i.e. activity at one point depends on the activity at neighboring points according to electromagnetic laws). The solution space was calculated on a realistic head model that included 4024 nodes, selected from a $6 \times 6 \times 6-\mathrm{mm}$ grid equally distributed within the gray matter of the Montreal Neurological Institute's average brain.

\section{RESULTS}

\section{Behavioral results}

Reaction times. Fig. 2a shows the mean RTs for the correct responses of all 11 participants in the upright and inverted conditions. There was a significant effect of Orientation $(F(1,10)=62.31, \quad P<0.001)$, with inverted stimuli (mean $\pm S D=1074.5 \pm 270.5 \mathrm{~ms}$; Supplemental Table 1a) eliciting longer RTs than upright stimuli (mean $\pm \mathrm{SD}=$ 764.0 $\pm 163.4 \mathrm{~ms}$; Supplemental Table 1b). We also found a main effect of Angle $(F(4,40)=7.8, P<0.001)$ with increasing RTs as the angle of rotation increased. Finally, a significant Orientation $\times$ Angle interaction $(F(4,40)=8.5, P<0.001)$ was found, reflecting the different RT patterns for upright and inverted stimuli: whereas RTs increased monotonically for the upright condition, they followed a U-shaped function for the inverted stimuli. In the upright condition, 10 of the 11 participants showed a monotonic increase with the increasing angle of rotation and in the inverted condition, 9 of the 11 participants displayed a U-shaped pattern.

Error rates. Fig. 2b shows the mean error rates in the upright and inverted conditions. The patterns were similar to those observed for reaction times. The $2 \times 5$ repeated measures ANOVA with Orientation (upright, inverted) and Angle $\left(0^{\circ}, 45^{\circ}, 90^{\circ}, 135^{\circ}, 180^{\circ}\right)$ as within-subject factors showed an effect of Orientation $(F(1,10)=12.1, P<0.01)$, revealing higher error rates for the inverted stimuli (mean $\pm S D=14.1 \% \pm 12.4 \%$; Supplemental Table 2b) compared to the upright ones (mean $\pm \mathrm{SD}=3.2 \% \pm 3.8 \%$; Supplemental Table 2a). Also, a main effect of Angle $(F(4,40)=8.6, P<0.001)$ was shown, with increasingly higher error rates for larger angles. Finally, we found a significant Orientation $\times$ Angle interaction $(F(4,40)=4.3, P<0.01)$, reflecting the different error patterns for upright and inverted

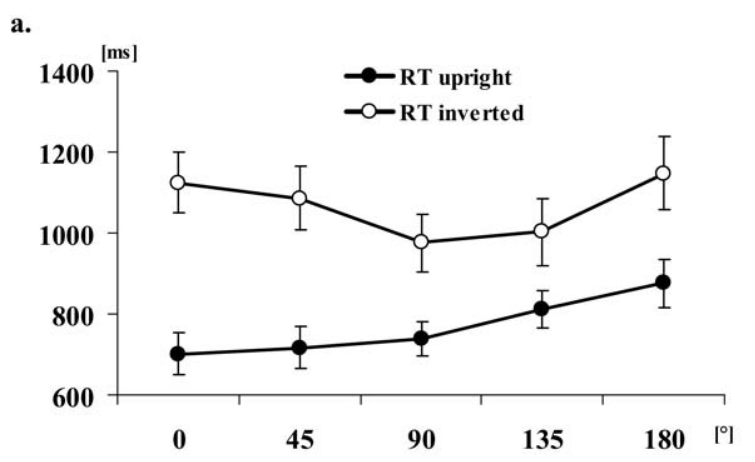

b.

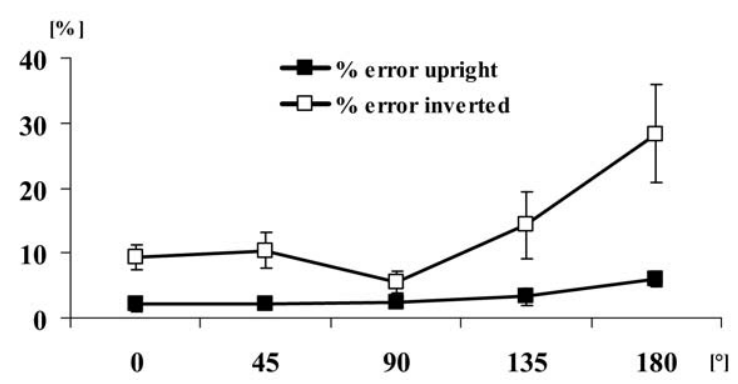

Fig. 2. (a) Behavioral results: upright and inverted stimulus conditions: Mean RTs [mean \pm SEM] as a function with the increasing angle of rotation $\left({ }^{\circ}\right)$. (b) Behavioral results: upright and inverted stimulus conditions: Mean error rates [mean \pm SEM] as a function with the increasing angle of rotation $\left({ }^{\circ}\right)$. Data combined for $45^{\circ}-315^{\circ}$, angles $135^{\circ}-225^{\circ}$, and angles $90^{\circ}-270$ as the results were similar for these sets of angles and they represented the same angle of rotation in opposite quadrants.

stimuli depending on the stimulus angle. As for RTs, errors for upright bodies increased monotonically, they followed a Ushaped function for the inverted stimuli (the latter was less obvious than for RTs, but was especially evident and significant for the two largest angles and $90^{\circ}$ ).

Participant reports. Debriefing of the participants after the experiments revealed that all 11 participants had imagined themselves in the position of the presented human figure before giving their answer. They also reported that upright human body positions were easier to adopt than inverted ones. 9 of the 11 participants stated that for upright stimuli some angles were easier to identify $\left(0^{\circ}, 45^{\circ}\right)$ than others $\left(180^{\circ}\right)$. However, this was not the case for inverted stimuli as participants did not particularly report some angles to be easier than others.

\section{EP analysis}

EP mapping of the group-averaged data revealed several distinct time segments of brain activity as reflected by stable voltage topography (or EP maps) for the upright and inverted condition. These brain activations showed differences depending on the angle of the body stimulus and whether mental body transformations were carried out for upright or inverted bodies. Our data reveal three such sequential steps of brain activation in the time periods of both upright and inverted conditions from $\sim 220$ to $\sim 490 \mathrm{~ms}$ after stimulus onset. First, brain activity from $\sim 220$ to $\sim 360$ ms (1) encodes 

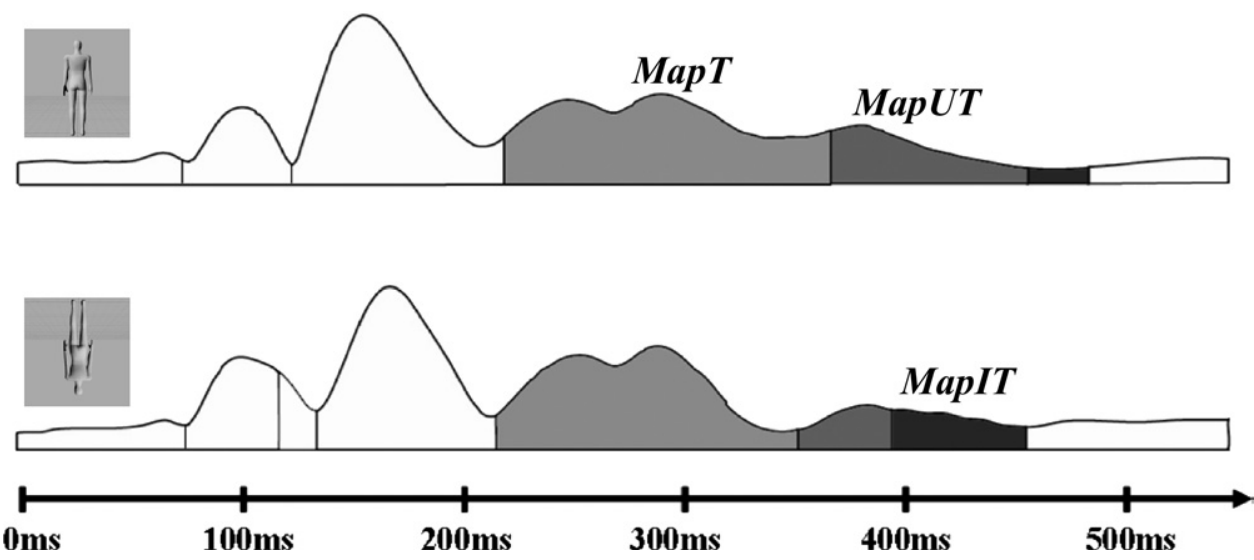

Fig. 3. EEG Results: grand mean ERPs over 11 participants for both the conditions (upright, inverted). Traces show the GFP (field strength) in microvolts $(\mu \mathrm{V})$ of the 192-channel recording as a function of time in milliseconds (stimulus onset at $0 \mathrm{~ms}$ ). Labels at the curves correspond to the segments: MapT, MapUT, Map/T (functional maps) found with the spatiotemporal segmentation method.

mental body transformation independent of whether the shown body is upright or inverted (MapT). Second, brain activity from $\sim 350$ to $\sim 460 \mathrm{~ms}$, encodes (2) mental body transformations for upright bodies (MapUT) followed $(\sim 390$ to $\sim 490 \mathrm{~ms}$ ) by brain activity encoding (3) mental body transformations for inverted bodies (Map/T; Fig. 3).

Brain activation at 220-360 ms encodes mental body transformation. Our analysis revealed the presence of brain activity (MapT), whose duration (in ms) and GFP (map strength in $\mu \mathrm{V}$ ) reflected behavioral performances for upright and inverted bodies. This is shown in Fig. 4, where we plotted duration and strength of MapT for upright bodies that correlate positively with Angle, but follow a Ushaped function for inverted bodies. MapT appeared from $\sim 220$ to $\sim 360 \mathrm{~ms}$ after visual stimulus onset (Fig. 3). For the duration of $M a p T$, statistical analysis revealed no effect of Orientation $(F(1,10)=0.32, P=0.58)$. There was no main effect of angle $(F(4,40)=2.17, P=0.09)$. No significant Orientation $\times$ Angle interaction was found $F(4,40)=0.97$, $P=0.43$ ). For the strength (or GFP) of MapT, statistical analysis revealed a main effect of Angle $(F(4,40)=3.2$, $P<0.05)$, with higher GFP for the larger angles than the smaller ones. Moreover, a significant Orientation $\times$ Angle $(F(4,40)=3.5, P<0.05)$ was found with GFP increasing linearly with angles in the case of upright bodies, but not in the case of inverted ones where it followed a U-shaped function (Fig. 4). No significant effect of Orientation was found $(F(1,10)=0.42, P=0.53)$. To summarize, the strength of MapT that ranges from $\sim 220$ to $\sim 360 \mathrm{~ms}$, closely reflects behavioral performance in mental body transformations for the different angles for upright as well as inverted bodies. This suggests that the strength, but not the duration, of MapT reflects the mental transformation effort differently for upright and inverted bodies.

Brain activation at 350-460 ms encodes mental body transformation for upright bodies. Following MapT, our analysis revealed the presence of MapUT that appeared between $\sim 350$ to $\sim 460 \mathrm{~ms}$ after visual stimulus onset (Fig. 3) and reflected mental body transformations for upright bodies. The duration and strength of MapUT inversely correlated with the angle of upright bodies (Fig. 5a). Thus, MapUT lasted longer for upright bodies (mean duration: $100.4 \pm 14.9 \mathrm{~ms}$ ) than for inverted bodies (mean duration: $50.0 \pm 16.1 \mathrm{~ms} ; F(1,10)=9.8, P<0.05)$ without showing any further effects with respect to its duration: Angle $F(4,40)=$ $0.74, P=0.56$; Orientation $\times$ Angle interaction $(F(4,40)=1.4$,

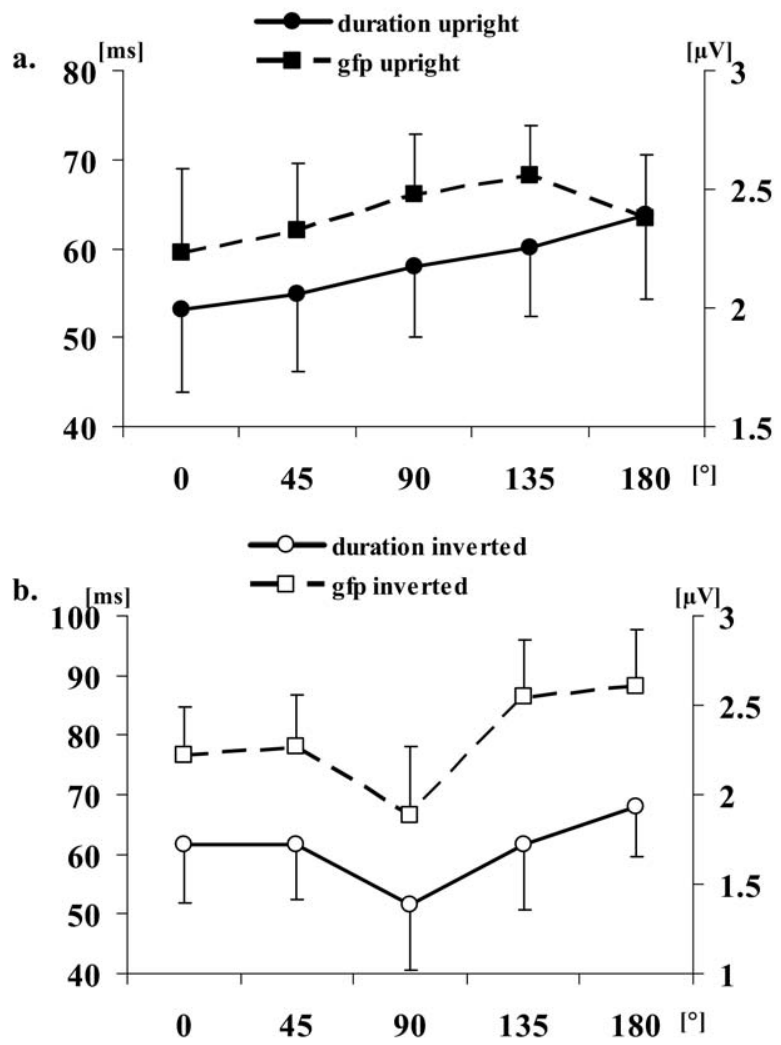

Fig. 4. EEG Results. (a) Mean duration (ms) and GFP in ( $\mu \mathrm{V})$ of MapT [mean \pm SEM] as a function with the increasing angle of rotation $\left(^{\circ}\right)$ in the upright condition. (b) Mean duration (ms) and GFP in $(\mu \mathrm{V})$ of MapT [mean \pm SEM] as a function with the increasing angle of rotation $\left(^{\circ}\right)$ in the inverted stimulus condition. 
a.

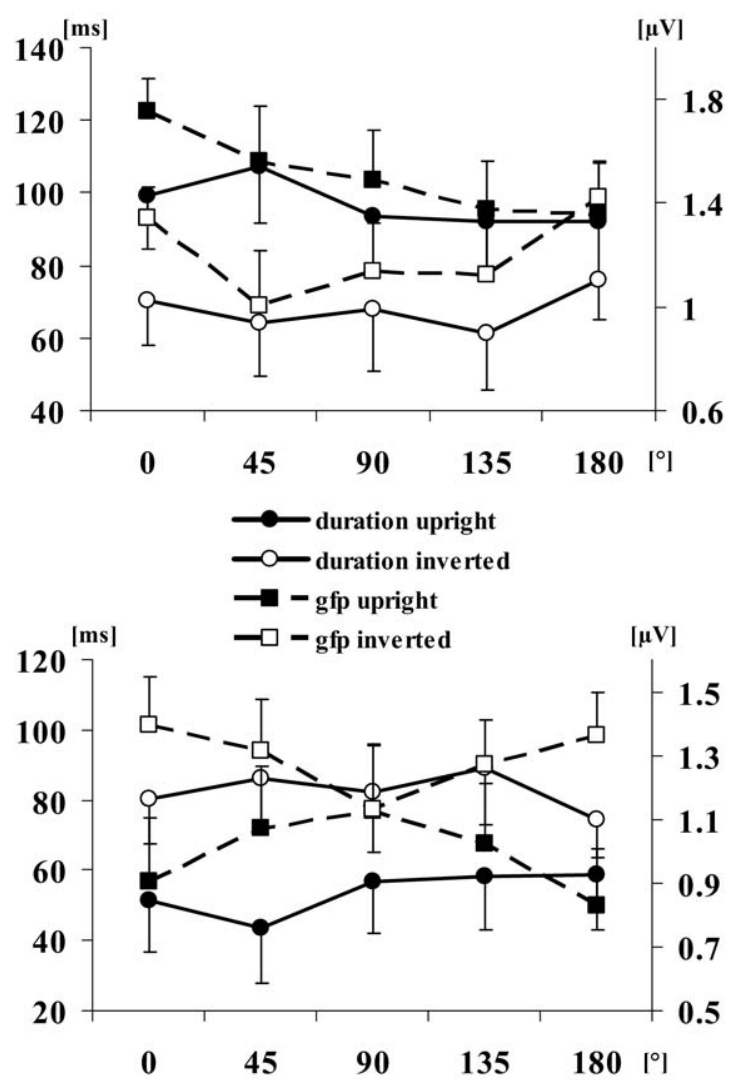

Fig. 5. Electrophysiology results. (a) Mean duration (ms) and GFP in $(\mu \mathrm{V})$ of MapUT [mean \pm SEM] as a function with the increasing angle of rotation $\left({ }^{\circ}\right)$ in the upright and the inverted stimulus condition. (b) Mean duration (ms) and GFP in $(\mu \mathrm{V})$ of Map/T [mean \pm SEM] as a function with the increasing angle of rotation $\left(^{\circ}\right)$ in the upright and the inverted stimulus condition.

$P=0.24)$. For the strength of MapUT, we found a significant main effect of Orientation $(F(1,10)=7.6, P<0.05)$, with higher GFP values for the upright than the inverted condition (Fig. 5) as well as a significant Orientation $\times$ Angle interaction $(F(4,40)=3.0, P<0.05)$, with GFP progressively decreasing with the angles of upright bodies (Fig. 5a), and a U-shaped function for the angles of inverted bodies (Fig. 5a). No main effect of Angle was found $(F(4,40)=1.7, P=0.17)$. Note that inspection of Fig. 5 a reveals similar patterns for the duration of MapUT, although these were not significant except for orientation. To summarize, MapUT appears from $\sim 350-$ $\sim 460 \mathrm{~ms}$, and is prolonged and stronger during mental trans- formations of upright bodies, and decreases in strength for upright bodies with increasing angles.

Brain activation at 390-490 ms encodes mental body transformation for inverted bodies. The following Map/T appeared between $\sim 390$ to $\sim 490$ ms after visual stimulus onset (Fig. 3) and reflected mental body transformations for inverted bodies. Its duration and strength were increased for inverted bodies and its strength mimicked behavioral performance (RTs) for inverted bodies (Fig. 5b). With respect to its duration, Map/T lasted significantly longer for inverted bodies (mean duration: 82.8 $\pm 16.1 \mathrm{~ms}$ ) than upright bodies (mean duration: 67.6 $\pm 14.9 \mathrm{~ms}$; $F(1,10)=9.8, P<0.05$; Fig. $5 b)$ without a significant main effect of Angle $(F(4,40)=0.74, P=0.56)$ or a significant Orientation $\times$ Angle interaction $(F(4,40)=1.4, P=0.24)$. For GFP strength we found a significant effect of Orientation $(F(1,10)=5.8, P<0.05)$, with higher GFP for inverted bodies as compared to upright bodies. Moreover, a significant Orientation $\times$ Angle interaction was found $(F(4,40)=4.7$, $P<0.01$ ), with GFP progressively decreasing from 0 to $90^{\circ}$ (Fig. 5b; empty squares) and then increasing from 90 to $180^{\circ}$ for inverted bodies (U-shape) (Supplemental Table $5 d$ ), while the opposite pattern was found for upright bodies (inverted U-shape; Fig. 5b. No main effect of Angle was found $(F(4,40)=0.25, P=0.90)$. To summarize, MaplT appears from $\sim 390$ to $\sim 490 \mathrm{~ms}$, is prolonged and stronger during mental transformations of inverted bodies and its strength mimics RTs for inverted bodies.

Electrical neuroimaging: source localization. A linear inverse solution (LAURA; Grave de Peralta et al., 2001, 2004) was applied to all three EP maps.

MapT was localized to bilateral temporo-occipital cortex and left temporo-parietal cortex. The maximal peak of activation in the left temporo-occipital cortex was at Talairach coordinates: $-36[\mathrm{x}],-75[\mathrm{y}]$, and $-12[\mathrm{z}]$; Fig. 6 . The maximal peak of activation in the right temporo-occipital cortex was localized at the Talairach coordinates; +47 $[\mathrm{x}],-58[\mathrm{y}]$, and $-11[\mathrm{z}]$. The maximal peak of activation in the left temporo-parietal cortex was localized to Talairach coordinates $-47[\mathrm{x}],-60[\mathrm{y}]$, and $+17[\mathrm{z}]$.

MapUT was localized to bilateral temporo-occipital cortex and the maximal peak of activation was in left temporooccipital cortex (Talairach coordinates; $-18[\mathrm{x}],-87$ [y], and $-13[\mathrm{z}])$. Other activated brain regions were in the vicinity of the left precuneus and/or the medial parieto-
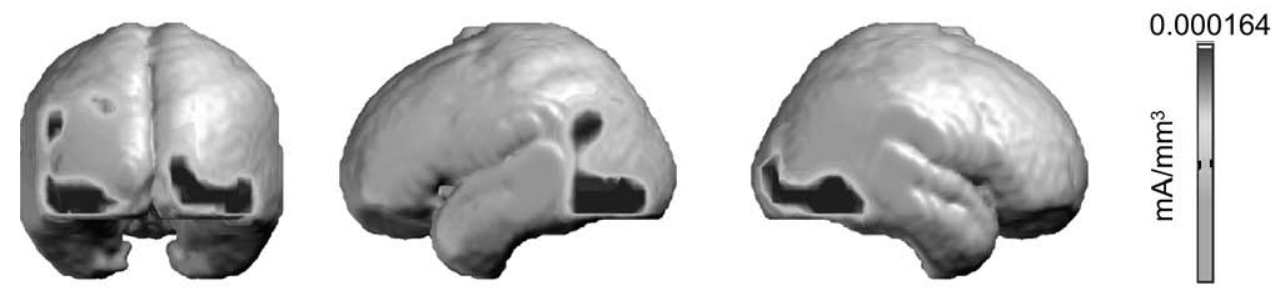

Fig. 6. Source localization. The generators of MapT were localized bilaterally to the temporo-occipital areas, and the left temporo-parietal cortex. 
a.

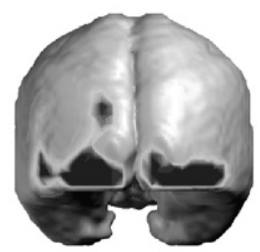

b.

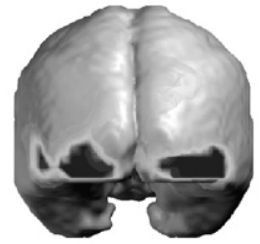

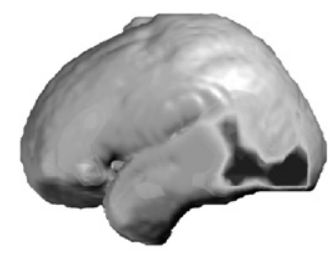

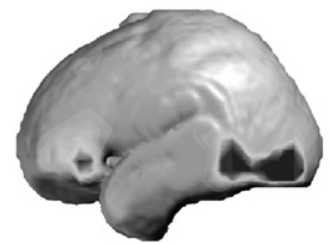

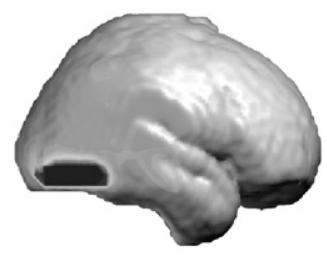

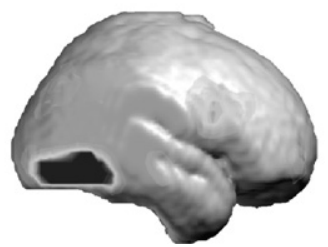

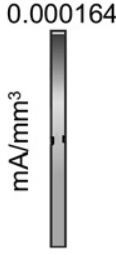

0

Fig. 7. Source Localization. (a) The generators of MapUT were localized to bilateral temporo-occipital lobes and in the vicinity of the left precuneus region. (b) The generators of Map/T were localized to bilateral occipital, slight temporal lobes, and left pre-frontal area.

occipital cortex (Talairach coordinates; $-18[\mathrm{x}],-83[\mathrm{y}]$, and $+20[z])$. This is shown in Fig. $7 a$.

Map/T was localized to bilateral temporo-occipital cortex and the maximal peak of activation was in right temporo-occipital cortex (Talairach coordinates; $+42[x],-69$ [y], and -12 [z]; Fig. 7b). The Talairach coordinates of the peak activation in the left temporo-occipital cortex, were $-24[\mathrm{x}],-81[\mathrm{y}]$, and $-12[\mathrm{z}]$. In addition, left ventral prefrontal cortex was activated (Talairach coordinates; $-42[\mathrm{x}],+35[\mathrm{y}]$, and $-2[\mathrm{z}])$.

\section{DISCUSSION}

The aim of this study was to investigate the time course and location of brain activation during the mental transformation of whole human bodies. A large set of stimulus angles (as used by Parsons (1987a) in his seminal study) employed and the body stimuli were presented in upright and inverted orientations. Behavioral analysis revealed two main findings: participants were faster for upright than inverted bodies and RTs were different depending on the angle of the bodily stimulus (and whether the body was upright or inverted). More specifically, RTs and error rates increased linearly with the angle of rotation for upright bodies, likely reflecting a mental transformation process, whereas the pattern of RTs and error rates was different for inverted bodies. This is compatible with data showing that the processing of bodies and body parts in the familiar upright position is facilitated and qualitatively different from the processing of bodies and body parts (arms, hands, and fingers) in unfamiliar or impossible positions (Parsons, 1987b; Parsons et al., 1995; Daems and Verfaillie, 1999; Verfaillie and Daems, 2002; Overney et al., 2005; Petit and Harris, 2005). We note that this "inversion effect" in mental imagery may also be comparable to the findings of Reed et al. (2003) regarding the inversion effect for human bodies during perceptual tasks. Reed et al. (2003) found that inverted bodies are processed slower and less accurately than upright bodies and this is arguably evidence for the configural processing of upright human bodies (Reed et al., 2003, 2006). At present it is not known whether the observed "inversion effect" in mental imagery is due to interference with configural mechanisms, incongruency between the participant's body position and the orientation of the stimulus, greater visual familiarity for upright bodies, or the impossibility of corresponding body positions. Likely, a combination of these different factors influences the mental imagery for these important social visual stimuli. In the current study, it is possible that participants used different rotation routes across the different angles of inverted stimuli. Participants could have used either the picture plane rotation for some stimulus angles as compared to the depth plane rotation for other angles. Rotation strategies in the roll plane have been reported to lead to slower RTs than rotation strategies in the pitch plane (Murray, 1997) and our participants may have used different strategies depending on the orientation and angle of the stimulus.

The main goal of the present neuroimaging study as mentioned above was to investigate the time course and location of brain activation during mental whole body transformations. Using EP mapping and electrical neuroimaging we show that such transformations are associated with three distinct processing steps: (1) an early brain activation that does not distinguish between upright and inverted bodies, but closely reflects the effort of mental transformation, followed by a brain activation that (2) encodes mental transformation for upright bodies, and a later brain activation that (3) encodes mental transformation for inverted bodies. These data suggest that mental body transformation is not a single process but a sequence of processing steps, each of which reflects a different aspect of the transformation process depending on rotation angle and/or stimulus orientation. Our ERP data further reveal that the differences in strength (and duration) between the experimental conditions during these three processing steps are relative and not absolute and accordingly also point to a functional overlap during all three sequences of brain processing.

The brain activity associated with mental transformation for upright and inverted bodies (1, MapT) appeared 
between 220 and 360 ms and was thus slightly earlier than what has previously been found for mental transformation or rotation of external objects (between 300 and 800 ms; Kosslyn et al., 1994; Pegna et al., 1997; Harris et al., 2000; Jordan et al., 2001; Gauthier and Tarr, 2002; Podzebenko et al., 2002; Harris and Miniussi, 2003). However, it has also been suggested that the mental transformation of human bodies may occur at an earlier latency than for other objects: $330 \mathrm{~ms}$ for human bodies (Blanke et al., 2005) and $310 \mathrm{~ms}$ for body parts (Overney et al., 2005). The present data are compatible with such slightly earlier brain processing related to mental transformation concerning human bodies, when tested with many angles and compared to inverted bodies. We also believe that the brain processes represented by MapT, UT, and IT are related to mental transformation and not simply to visual perception as ERP components related to visual perception are generally found earlier-at $\sim 70,100$, or $\sim 170 \mathrm{~ms}$ after stimulus onset-as compared to the present ERP components (Itier et al., 2004; Itier and Taylor, 2004; Stekelenburg and de Gelder, 2004; Thierry et al., 2006; Meeren et al., 2008). The timing of the brain activations in the present experiment also conforms with the time periods found in previous studies on mental imagery from us and other groups (Pegna et al., 1997; Jordan et al., 2001; Harris and Miniussi, 2003; Milivojevic et al., 2003; Blanke et al., 2005; Overney et al., 2005; Arzy et al., 2006). Finally, previous work has also carried out "passive viewing" control conditions (without mental imagery) and described the absence of the ERP processes linked to mental body transformation at $\sim 300-450 \mathrm{~ms}$ (e.g. Blanke et al., 2005 , as presented here). Concerning the neural mechanisms, previous studies on mental rotation of external objects have shown bilateral occipito-parietal, and frontal activations, whereas body transformations have been linked to activations in the bilateral temporo-parietal cortex, posterior parietal and the extrastriate cortex. It thus seems that mental transformation processes for body and nonbody stimuli rely at least partly on distinct (as well as shared) brain mechanisms with respect to the neuroanatomy and the timing of brain activity. Of course this statement has to be regarded with caution, since the present study did not (and did not intend to) compare mental transformation of bodies versus body parts or other objects. It will be important in future work to determine the exact degree of spatial and temporal overlap of brain activations related to the mental transformation of whole bodies and other objects. The strength of the early brain activation MapT closely reflected the behavioral mental transformation curves for upright as well as inverted bodies. Accordingly, we suggest that this brain activity represents the aspect of the mental transformation process that encodes the mental activity related to both upright and inverted stimulus orientations. Our finding of brain activations in the temporo-parietal cortex and the extrastriate cortex is compatible with the results of earlier studies using EP mapping and electrical neuroimaging (Blanke et al., 2005; Arzy et al., 2007) and fMRI (Zacks et al., 1999). The activation is also partly overlapping with or adjacent to the posterior parietal cortex, and activation in this region has previously been observed in several mental transformation studies (Tagaris et al., 1996; Alivisatos and Petrides, 1997; Richter et al., 1997, 2000; Carpenter et al., 1999; Iwaki et al., 1999; Harris et al., 2000; Jordan et al., 2001; Podzebenko et al., 2002).

For the period from $\sim 350$ to $\sim 490 \mathrm{~ms}$ after stimulus onset, our electrophysiological data reveal a double dissociation between mental transformation for (2) upright and (3) inverted bodies, both being associated with a different brain activation. These periods were partly overlapping, but different time periods for the mental transformation of upright bodies (350-460 ms) and inverted bodies (390$490 \mathrm{~ms}$ ) were found. Thus, whereas brain activation reflected by MapUT (stage 2) was longer lasting and stronger in the upright than the inverted condition, the opposite was true for the brain activation reflecting Map/T (stage 3). As in all previous work from our group as well as other ERP data these dissociations or double dissociations are relative rather than absolute. Moreover, both brain activations did not simply dissociate between stimulus orientations, but also reflected transformation processes for their preferred stimulus: the strength and duration of MapUT correlated negatively with upright angles and RTs, whereas the strength and duration of Map/T correlated positively with RTs for inverted bodies. This suggests that these processes are complementary and more specific mental transformation processes compared to those reflected by the preceding brain activation (MapT). While both maps strongly activated extrastriate cortex, each of these maps also activated different regions in the brain. While MapUT showed additional activation of the medial parieto-occipital and temporo-parietal cortex (similar to MapT), Map/T was localized to the extrastriate and the left ventral prefrontal cortex (Stekelenburg and de Gelder, 2004). Activation of the medial parieto-occipital cortex in the upright condition (MapUT) is consistent with other findings on mental transformations highlighting posterior parietal activation (Alivisatos and Petrides, 1997; Richter et al., 1997, 2000; Iwaki et al., 1999; Harris et al., 2000; Jordan et al., 2001), and with a previous study on body part transformations using EP mapping and electrical neuroimaging (Overney et al., 2005). The temporo-parietal activation also corroborates previous data using the same EP mapping and electrical neuroimaging methods as employed in the present study (Blanke et al., 2005; Arzy et al., 2006). The prefrontal activation related to Map/T that we observed only in the case of inverted bodies also agrees with previous reports which suggests that this activation may be due to a surprise-related reaction or arousal while seeing unfamiliar bodily orientations (Overney et al., 2005) or bodily conflict (Fink et al., 2000; Tsakiris et al., 2007). The timing of MapUT and Map/T also confirms results from previous EP studies (Blanke and Arzy, 2005; Blanke et al., 2005; Arzy et al., 2006).

In summary, brain activity during all three processing steps—ranging from $\sim 220$ to $\sim 490 \mathrm{~ms}$ - encoded mental transformation processes for whole human bodies. Although the earliest brain activation in extrastriate and tem- 
poro-parietal cortex did not distinguish between transformations for upright and inverted bodies, both later brain activations did, suggesting that general mental transformation processes precede more specialized processes for whole human bodies, with those for inverted bodies being the most delayed. We reiterate that the present data does not allow us to claim that this sequence of processing steps is specific for the mental transformation of human bodies as opposed to other objects. This was the focus of previous work from us (Blanke et al., 2005; Overney and Blanke, 2009) as well as other groups (i.e. Kosslyn et al., 1998). As stated above, the main goal of the present neuroimaging study was to investigate the time course and location of brain activation during mental whole body transformations. The time period of both later brain activations (MapUT and Map/T) overlaps with the time period of brain activations reflecting other dissociations in mental body transformations for whole bodies, such as embodied versus disembodied self locations (Blanke and Arzy, 2005; Arzy et al., 2006), and mental transformation of external objects, suggesting the involvement of this later period in more specific (i.e. upright bodies, inverted bodies, hands, external objects, self) transformation processes, whereas the earlier activity is likely to reflect more basic aspects of mental transformations. These data suggest that cogitations (spatial reasoning, action planning and problem solving as measured by mental imagery; Shepard and Hurwitz, 1984; Corballis, 1997) with respect to one of the most frequent visual stimuli in our social world are encoded by a sequence of brain activations in a specific time window and specific brain areas. We note, that this statement has to be regarded as preliminary since we did not test the mental transformation of any stimuli other than whole human bodies. In future work it will also be important to determine the degree of spatial and temporal overlap of brain activations related to the perception of whole bodies (Allison et al., 2000; Downing et al., 2001; Thierry et al., 2006; Urgesi et al., 2007) and those cogitations related to the mental transformations of these stimuli.

\section{REFERENCES}

Alivisatos B, Petrides M (1997) Functional activation of the human brain during mental rotation. Neuropsychologia 35:111-118.

Allison T, Puce A, McCarthy G (2000) Social perception from visual cues: role of the STS region. Trends Cogn Sci 4:267-278.

Arzy S, Mohr C, Michel CM, Blanke O (2007) Duration and not strength of activation in temporo-parietal cortex positively correlates with schizotypy. Neuroimage 35:326-333.

Arzy S, Thut G, Mohr C, Michel CM, Blanke O (2006) Neural basis of embodiment: distinct contributions of temporoparietal junction and extrastriate body area. J Neurosci 26:8074-8081.

Astafiev SV, Stanley CM, Shulman GL, Corbetta M (2004) Extrastriate body area in human occipital cortex responds to the performance of motor actions. Nat Neurosci 7:542-548.

Blanke O, Mohr C, Michel CM, Pascual-Leone A, Brugger P, Seeck M, Landis T, Thut G (2005) Linking out-of-body experience and self processing to mental own-body imagery at the temporoparietal junction. J Neurosci 25:550-557.

Bonda E, Frey S, Petrides M (1996) Evidence for a dorso-medial parietal system involved in mental transformations of the body. J Neurophysiol 76:2042-2048.
Carpenter PA, Just MA, Keller TA, Eddy W, Thulborn K (1999) Graded functional activation in the visuospatial system with the amount of task demand. J Cogn Neurosci 11:9-24.

Cohen MS, Kosslyn SM, Breiter HC, DiGirolamo GJ, Thompson WL, Anderson AK, Brookheimer SY, Rosen BR, Belliveau JW (1996) Changes in cortical activity during mental rotation. A mapping study using functional MRI. Brain 119 (Pt 1):89-100.

Cooper LA, Shepard RN (1975) Mental transformations in the identification of left and right hands. J Exp Psychol Hum Percept Perform 104:48-56.

Corballis MC (1997) Mental rotation and the right hemisphere. Brain Lang 57:100-121.

Daems A, Verfaillie K (1999) Viewpoint-dependent priming effects in the perception of human actions and body postures. Vis Cogn 6:665-693.

Downing PE, Jiang Y, Shuman M, Kanwisher N (2001) A cortical area selective for visual processing of the human body. Science 293:2470-2473.

Fink GR, Marshall JC, Weiss PH, Shah NJ, Toni I, Halligan PW, Zilles K (2000) "Where" depends on "what": a differential functional anatomy for position discrimination in one-versus two-dimensions. Neuropsychologia 38:1741-1748.

Fiorio M, Tinazzi M, Ionta S, Fiaschi A, Moretto G, Edwards MJ, Bhatia KP, Aglioti SM (2007) Mental rotation of body parts and noncorporeal objects in patients with idiopathic cervical dystonia. Neuropsychologia 45:2346-2354.

Ganis G, Keenan JP, Kosslyn SM, Pascual-Leone A (2000) Transcranial magnetic stimulation of primary motor cortex affects mental rotation. Cereb Cortex 10:175-180.

Gauthier I, Tarr MJ (2002) Unraveling mechanisms for expert object recognition: bridging brain activity and behavior. J Exp Psychol Hum Percept Perform 28:431-446.

Grave de Peralta Menendez R, Gonzalez Andino S, Lantz G, Michel CM, Landis T (2001) Noninvasive localization of electromagnetic epileptic activity. I. Method descriptions and simulations. Brain Topogr 14:131-137.

Grave de Peralta Menendez R, Murray MM, Michel CM, Martuzzi R, Gonzalez Andino SL (2004) Electrical neuroimaging based on biophysical constraints. Neuroimage 21:527-539.

Harris IM, Egan GF, Sonkkila C, Tochon-Danguy HJ, Paxinos G, Watson JD (2000) Selective right parietal lobe activation during mental rotation: a parametric PET study. Brain 123 (Pt 1):65-73.

Harris IM, Miniussi C (2003) Parietal lobe contribution to mental rotation demonstrated with rTMS. J Cogn Neurosci 15:315-323.

lonta S, Fourkas AD, Fiorio M, Aglioti SM (2007) The influence of hands posture on mental rotation of hands and feet. Exp Brain Res 183:1-7.

Itier RJ, Taylor MJ (2004) Source analysis of the N170 to faces and objects. Neuroreport 15:1261-1265.

Itier RJ, Taylor MJ, Lobaugh NJ (2004) Spatiotemporal analysis of eventrelated potentials to upright, inverted, and contrast-reversed faces: effects on encoding and recognition. Psychophysiology 41:643-653.

Iwaki S, Ueno S, Imada T, Tonoike M (1999) Dynamic cortical activation in mental image processing revealed by biomagnetic measurement. Neuroreport 10:1793-1797.

Jordan K, Heinze HJ, Lutz K, Kanowski M, Jancke L (2001) Cortical activations during the mental rotation of different visual objects. Neuroimage 13:143-152.

Keenan JP, Nelson A, O'Connor M, Pascual-Leone A (2001) Selfrecognition and the right hemisphere. Nature 409:305.

Kosslyn SM, Alpert NM, Thompson WL, Chabris CF, Rauch SL, Anderson AK (1994) Identifying objects seen from different viewpoints. A PET investigation. Brain 117 (Pt 5):1055-1071.

Kosslyn SM, DiGirolamo GJ, Thompson WL, Alpert NM (1998) Mental rotation of objects versus hands: neural mechanisms revealed by positron emission tomography. Psychophysiology 35:151-161.

Lehmann D, Ozaki H, Pal I (1987) EEG alpha map series: brain micro-states by space-oriented adaptive segmentation. Electroencephalogr Clin Neurophysiol 67:271-288. 
Lehmann D, Skrandies W (1980) Reference-free identification of components of checkerboard-evoked multichannel potential fields. Electroencephalogr Clin Neurophysiol 48:609-621.

Meeren HK, Hadjikhani N, Ahlfors SP, Hämäläinen MS, de Gelder B (2008) Early category-specific cortical activation revealed by visual stimulus inversion. PLoS ONE 3(10):e3503.

Michel CM, Grave de Peralta R, Lantz G, Gonzalez Andino S, Spinelli L, Blanke O, Landis T, Seeck M (1999) Spatiotemporal EEG analysis and distributed source estimation in presurgical epilepsy evaluation. J Clin Neurophysiol 16:239-266.

Michel CM, Murray MM, Lantz G, Gonzalez S, Spinelli L, Grave de Peralta R (2004) EEG source imaging. Clin Neurophysiol 115: 2195-2222.

Michel CM, Thut G, Morand S, Khateb A, Pegna AJ, Grave de Peralta R, Gonzalez S, Seeck M, Landis T (2001) Electric source imaging of human brain functions. Brain Res Brain Res Rev 36:108-118.

Milivojevic B, Johnson BW, Hamm JP, Corballis MC (2003) Nonidentical neural mechanisms for two types of mental transformation: event-related potentials during mental rotation and mental paper folding. Neuropsychologia 41:1345-1356.

Murray JE (1997) Flipping and spinning: spatial transformation procedures in the identification of rotated natural objects. Mem Cognit 25:96-105.

Overney LS, Michel CM, Harris IM, Pegna AJ (2005) Cerebral processes in mental transformations of body parts: recognition prior to rotation. Brain Res Cogn Brain Res 25:722-734.

Overney LS, Blanke O (2009) Impaired imagery for upper limbs. Brain Topogr. http://www.ncbi.nlm.nih.gov/pubmed/18800243. (In press).

Parsons LM (1987a) Imagined spatial transformation of one's body. J Exp Psychol Gen 116:172-191.

Parsons LM (1987b) Imagined spatial transformations of one's hands and feet. Cognit Psychol 19:178-241.

Parsons LM (1994) Temporal and kinematic properties of motor behavior reflected in mentally simulated action. J Exp Psychol Hum Percept Perform 20:709-730.

Parsons LM, Fox PT, Downs JH, Glass T, Hirsch TB, Martin CC, Jerabek PA, Lancaster JL (1995) Use of implicit motor imagery for visual shape discrimination as revealed by PET. Nature 375:54-58.

Parsons LM, Gabrieli JD, Phelps EA, Gazzaniga MS (1998) Cerebrally lateralized mental representations of hand shape and movement. J Neurosci 18:6539-6548.

Pascual-Marqui RD, Michel CM, Lehmann D (1995) Segmentation of brain electrical activity into microstates: model estimation and validation. IEEE Trans Biomed Eng 42:658-665.

Pegna AJ, Khateb A, Spinelli L, Seeck M, Landis T, Michel CM (1997) Unraveling the cerebral dynamics of mental imagery. Hum Brain Mapp 5:410-421.

Peronnet F, Farah MJ (1989) Mental rotation: an event-related potential study with a validated mental rotation task. Brain Cogn 9:279-288.

Petit LS, Harris IM (2005) Anatomical limitations in mental transformations of body parts. Vis Cogn 2(5):737-758.

Petit LS, Pegna AJ, Mayer E, Hauert CA (2003) Representation of anatomical constraints in motor imagery: mental rotation of a body segment. Brain Cogn 51:95-101.

Podzebenko K, Egan GF, Watson JD (2002) Widespread dorsal stream activation during a parametric mental rotation task, revealed with functional magnetic resonance imaging. Neuroimage 15:547-558.

Reed CL, Stone VE, Bozova S, Tanaka J (2003) The body-inversion effect. Psychol Sci 14:302-308.

Reed CL, Stone VE, Grubb JD, McGoldrick JE (2006) Turning configural processing upside down: part and whole body postures. J Exp Psychol Hum Percept Perform 32:73-87.
Richter W, Somorjai R, Summers R, Jarmasz M, Menon RS, Gati JS, Georgopoulos AP, Tegeler C, Ugurbil K, Kim SG (2000) Motor area activity during mental rotation studied by time-resolved single-trial fMRI. J Cogn Neurosci 12:310-320.

Richter W, Ugurbil K, Georgopoulos A, Kim SG (1997) Time-resolved fMRI of mental rotation. Neuroreport 8:3697-3702.

Sekiyama K (1982) Kinesthetic aspects of mental representations in the identification of left and right hands. Percept Psychophys 32:89-95.

Shepard RN, Hurwitz S (1984) Upward direction, mental rotation, and discrimination of left and right turns in maps. Cognition 18: 161-193.

Shepard RN, Metzler J (1971) Mental rotation of three-dimensional objects. Science 171:701-703.

Stekelenburg JJ, de Gelder B (2004) The neural correlates of perceiving human bodies: an ERP study on the body-inversion effect. Neuroreport 15:777-780.

Sugiura M, Watanabe J, Maeda Y, Matsue Y, Fukuda H, Kawashima $R$ (2005) Cortical mechanisms of visual self-recognition. Neuroimage 24:143-149.

Tagaris GA, Kim SG, Strupp JP, Andersen P, Ugurbil K, Georgopoulos AP (1996) Quantitative relations between parietal activation and performance in mental rotation. Neuroreport 7:773-776.

Tagaris GA, Richter W, Kim SG, Georgopoulos AP (1997) Box-Jenkins intervention analysis of functional magnetic resonance imaging data. Neurosci Res 27:289-294.

Thayer ZC, Johnson BW, Corballis MC, Hamm JP (2001) Perceptual and motor mechanisms for mental rotation of human hands. Neuroreport 12:3433-3437.

Thierry G, Pegna AJ, Dodds C, Roberts M, Basan S, Downing P (2006) An event-related potential component sensitive to images of the human body. Neuroimage 32:871-879.

Tsakiris M, Hesse MD, Boy C, Haggard P, Fink GR (2007) Neural signatures of body ownership: a sensory network for bodily selfconsciousness. Cereb Cortex 17:2235-2244.

Urgesi C, Candidi M, lonta S, Aglioti SM (2007) Representation of body identity and body actions in extrastriate body area and ventral premotor cortex. Nat Neurosci 10:30-31.

Verfaillie K, Daems A (2002) Representing and anticipating human actions in vision. Vis Cogn 9:217-232.

Vingerhoets G, Santens P, Van Laere K, Lahorte P, Dierckx RA, De Reuck J (2001) Regional brain activity during different paradigms of mental rotation in healthy volunteers: a positron emission tomography study. Neuroimage 13:381-391.

Wijers AA, Otten LJ, Feenstra S, Mulder G, Mulder LJ (1989) Brain potentials during selective attention, memory search, and mental rotation. Psychophysiology 26:452-467.

Yoshino A, Inoue M, Suzuki A (2000) A topographic electrophysiologic study of mental rotation. Brain Res Cogn Brain Res 9:121-124.

Zacks J, Rypma B, Gabrieli JD, Tversky B, Glover GH (1999) Imagined transformations of bodies: an $\mathrm{fMRI}$ investigation. Neuropsychologia 37:1029-1040.

Zacks JM, Michelon P (2005) Transformations of visuospatial images. Behav Cogn Neurosci Rev 4:96-118.

Zacks JM, Ollinger JM, Sheridan MA, Tversky B (2002) A parametric study of mental spatial transformations of bodies. Neuroimage 16:857-872.

\section{APPENDIX}

\section{Supplementary data}

Supplementary data associated with this article can be found, in the online version, at doi:10.1016/j.neuroscience.2009.02.012. 